Reproductive evolution

\section{Mating types and uniparental transmission of chloroplast genes}

\author{
from Brian Charlesworth
}

MANY sexually reproducing species of lower eukaryotes are not differentiated into males and females: all gametes are of equal size. Nevertheless, in a large number of species, gametes can be classified into different mating types, such that successful sexual fusion occurs only between cells of opposite mating type. Such species are called heterothallic, in contrast to homothallic species where no such restrictions on gametic union exist. The common pattern in both algae and lower fungi is to have just two mating types, controlled by alternative mendelian alleles ${ }^{1,2}$. In higher fungi (Basidiomycetes), many mating types, with more complex inheritance, may be present ${ }^{2}$. Mating types present an interesting problem for the evolutionary theorist. An ancillary problem is posed by the association of mating types with uniparental transmission of chloroplast genes in the green algae Chlamydomonas ${ }^{3}$, described in detail below.

The only serious theoretical study of the evolution of mating types has recently been made by Rolf Hoekstra ${ }^{4}$. He assumes a life cycle characteristic of a yeast or unicellular alga such as Chlamydomonas, in which haploid cells proliferate vegetatively by mitotic cell divisions. In appropriate conditions, the vegetative cells can differentiate into gametes, pairs of which can then fuse to form diploid zygotes. The zygotes undergo meiosis to produce tetrads of haploid daughter cells, which resume the cycle of vegetative growth. Hoekstra assumes that the species is initially homothallic, so that gametic union can occur between cells derived by vegetative division from the same meiotic product. Gamete recognition and fusion depends on (at least) two complementary factors, such as a diffusible pheromonal attractant and a corresponding receptor site. A gamete of a homothallic species produces both factors, and might be less efficient at recognition than gametes specialized to produce just one factor, because of self-saturation of the receptor sites. A mutant allele that, say, abolishes production of the pheromone might therefore have a selective advantage and invade the homothallic population, but clearly it cannot spread to fixation. Its presence would facilitate the spread of a mutant at another locus which abolishes production of the receptor sites. Selection would favour tighter linkage between the two loci, leading ultimately to the existence of a supergene involving variation of at least two distinct but tightly linked loci, with two complementary types of gamete predominating in the population. This prediction of a supergene controlling mating types agrees with genetic data on Chlamydomonas ${ }^{5}$, and with both genetic and molecular data on yeasts ${ }^{2,6-8}$. The model works best if the gamete recognition depends in part on diffusible pheromones; the mating-type loci in yeast are known to control such pheromones ${ }^{2,9}$.

This model thus provides an attractive explanation for heterothallism, at least for the case of two mating types, but how does one explain the existence of homothallic species? Homothallism would be favoured by selection in environments where population density is low, so that the chance of encounter is small for gametes derived from different vegetative clones. (This is analogous to the selection pressure towards self-fertilization observed in flowering plant species in which pollination efficiency is low ${ }^{10}$.) Homothallism in yeast is achieved by an elaborate mechanism in which both sorts of mating-type gene are present within each cell, one of the two being expressed as a result of movement to a special chromosomal site ${ }^{11}$. In this way, fusions between gametes derived from the same clone are made possible. There is also indirect evidence for the presence of two mating-type genes in the homothallic alga Chlamydomonas monoica ${ }^{12}$. This system is most easily understood in terms of des. cent from an ancestor that was originally heterothallic.

As mentioned earlier, mating types in Chlamydomonas raise another evolutionary issue. Denoting the mating types in heterothallic species as $m t^{+}$and $m t^{-}$, it was discovered by Ruth Sager in 1954 that certain mutant alleles are only transmitted to the progeny of a sexual cross when carried in the $m t^{+}$parent ${ }^{3}$. It now seems established that such mutants are carried on the chloroplast DNA ${ }^{13,14}$ and that the chloroplast DNA from the $m t^{-}$parent is destroyed in the zygote shortly after mating ${ }^{15,16}$. The chloroplast DNA in the progeny thus comes only from the $m t^{+}$ parent and is apparently protected from degradation by methylation of its cytosine residues ${ }^{16}$. This is in accordance with an hypothesis of Sager and Ramanis ${ }^{17}$. UV irradiation of the $\mathrm{mt}^{+}$gametes before mating reduces the frequency of uniparental transmission, showing that the effect of the $m t^{+}$locus is exerted before zygote formation ${ }^{18}$.

A new twist to the story has been provided by the work of Van Winkle-Swift and Aubert ${ }^{12}$ on the homothallic species Chlamydomonas monoica. In crosses between wild type and a mutant conferring resistance to erythromycin $(e r y-u l)$, they find that tetrads produced at meiosis show either 100 per cent transmission of ery-ul or 100 per cent transmission of wild type, each type of tetrad occurring with roughly equal frequency. They suggest that haploid cells of C.monoica contain both $\mathrm{mt}^{+}$and $m t^{-}$genes, but a gamete expresses only one of these, with fertile unions occurring solely between $m t^{+}$and $m t^{-}$. Uniparental inheritance follows the usual mating-type rule, accounting for the two classes of tetrad.

The evolutionary raison d'être of this convoluted mechanism of transmission of chloroplast DNA has been somewhat obscure, although Sager ${ }^{19}$ has suggested that it has the advantage of suppressing recombination between chloroplast genes. A simple explanation in terms of 'selfish DNA' is as follows. Consider a heterothallic species in which $\mathrm{mt}^{+}$and $\mathrm{mt}^{-}$ chloroplast genomes are transmitted in equal frequency to the next generation. A chloroplast mutation arises which interacts with a product of the mating-type region, in such a way that it causes destruction of the $m t^{-}$-derived chloroplast DNA when transmitted via an $\mathrm{mt}^{+}$gamete. Such a mutation will spread to fixation, since it ensures the exclusion from the progeny of chloroplast genomes derived from the opposite parent. A similar mechanism will operate in homothallic species, if Van Winkle-Swift and Aubert's model is correct and provided that at least some matings take place between gametes derived from different meiotic products. A difficulty is that two functions are required for uniparental transmission: protection of the $m t^{+}$-derived DNA and destruction of unprotected DNA. The postulated variant would thus have to involve a double mutational event. A similar requirement for two mutations exists for systems of unequal transmission of nuclear genes in other organisms ${ }^{20}$.

Brian Charlesworth is in the School of Biological Sciences, University of Sussex, Falmer, Brighton, Sussex BNI 9QG.

1. Lcwin, R.A. The Genetics of Algae (Blackwell, Oxford, 1976).

2. Fincham, J.R.S., Day, P.R. \& Radford, A. Fungal Genetics 4th edn (Blackwell, Oxford, 1979).

3. Sager, R. Proc. natn. Acad. Sci. U.S.A. 40, 356 (1954).

4. Hockstra, R. J. theor. Biol. 98,427 (1982).

5. Giltham, N.W. Am.Nat. 103, 355 (1969).

6. Klar, A.J.S. et al. Nature 289, 239 (1981).

I.cupoid, U. Cold Spring Harb. Symp. quant. Biol. 23, 161 (1958).

Meadc, J.H. \& Gutz, H. Genetics 83, 259 (1976).

9. Wilkinson, L.E. \& Pringle, J.R. Expl Cell Res, 89, 175 (1974).

10. Lloyd, D.G. in Demographic Factors and Mating Patterns in Angiosperms (ed. Solbrig, O.T.) (Blackwell, Oxford, 1980).

11. Hicks, J., Strathern, J.N. \& Klar, A.J.S. Nature 282. 478 (1979).

12. Van Winkle-Swift, K.P. \& Aubcrt, P. Nature 303, 167 (1983).

13. Spritzer, R.J. \& Mets, I..J. Nature 285, 114 (1980).

14. Grant, D.M., Gillham, N.W. \& Boynton, J.E. Proc. natn. Acad. Sci. U.S.A. 77, 6067 (1980).

15. Kuroiwa, T. et al. Nature 298, 481 (1982).

16. Burton, W.G.. Grabowy, C.T. \& Sager, R. Proc. natn. Acad. Sci. U.S. A. 76, 1390 (1979).

17. Sager, R.\& Ramanis, Z. Theor. appl. Genel. 43, 101 (1973).

18. Sager, R. \& Ramanis, Z.. Proc. natn. Acad. Sci. U.S.A. 58, 931 (1967).

19. Sager, R. in Genetics and Biogenesis of Mitochondria and Chloroplasts (eds Birky, C. W., Periman, P. S. \& Buyers, T. J.) 252 (Ohio State University Press, 1975). 20. Charlesworth, B., \& Harll, D.I. Genetics 89, 171 (1978) 